\title{
Capacité d'innovation des entreprises agroalimentaires et insertion dans les réseaux : le rôle de la proximité organisationnelle
}

Innovation capacity of agro-food firms and insertion in networks: the role of organisational proximity

Michel Martin, Corinne Tanguy et Pierre Albert

\section{(2) OpenEdition}

\section{Journals}

Édition électronique

URL : http://journals.openedition.org/economierurale/740

DOI : 10.4000/economierurale.740

ISSN : 2105-2581

\section{Éditeur}

Société Française d'Économie Rurale (SFER)

Édition imprimée

Date de publication : 2 mai 2006

Pagination : 35-49

ISSN : 0013-0559

Référence électronique

Michel Martin, Corinne Tanguy et Pierre Albert, « Capacité d'innovation des entreprises agroalimentaires et insertion dans les réseaux : le rôle de la proximité organisationnelle », Économie rurale [En ligne], 292 | Mars-avril 2006, mis en ligne le 05 mai 2008, consulté le 02 mai 2019. URL : http://journals.openedition.org/economierurale/740; DOI : 10.4000/economierurale.740 


\section{Capacité d'innovation des entreprises agroalimentaires et insertion dans les réseaux : le rôle de la proximité organisationnelle}

Michel MARTIN, Corinne TANGUY, Pierre ALBERT • INRA, Unité mixte de recherche, Centre d'économie et sociologie appliquées à l'agriculture et aux espaces ruraux, CESAER, Dijon

\section{Introduction}

L question de l'acquisition de compétences et de connaissances grâce à l'insertion des entreprises dans des réseaux est aujourd'hui considérée comme un enjeu primordial, tout particulièrement lorsque les entreprises n'ont pas acquis de capacités propres en Recherche-Développement (R\&D). Pourtant le fait que la très grande majorité des PME agroalimentaires dispose en interne d'un potentiel de R\&D très limité, voire inexistant, entraîne des difficultés à se mettre en contact avec des compétences technologiques externes. En matière de réseaux, il existe en réalité une imbrication forte entre le type d'environnement externe de l'entreprise et l'organisation interne. En ce qui concerne l'environnement externe de l'entreprise, il faut prendre en compte deux éléments indissociables :

- une partie correspond à une donnée pour l'entreprise : environnement institutionnel d'aide à l'innovation et au transfert, types d'organismes présents, collectivités locales, contrat de plan État-Région, etc. - une partie est construite par l'entreprise : partenariats privilégiés, mode de relations avec les partenaires, type de coordination avec les autres entreprises...

Dans cet article, nous tentons d'apporter des réponses à la question de l'ancrage territorial (Zimmermann, 1998) ${ }^{1}$ des entreprises, et particulièrement des PME, en examinant les relations que tissent les entreprises bourguignonnes avec leur envi- ronnement dans le cadre de leur activité d'innovation. La logique dans laquelle nous nous situons est une logique de coconstruction du territoire et de la firme, les performances des entreprises trouvant leurs fondements non seulement dans leurs caractéristiques propres mais également dans la qualité de leurs interactions avec l'ensemble des acteurs externes (entreprises, clients, centres techniques, scientifiques, centres de formation, etc.) Notre analyse est en fait centrée sur l'entreprise et sa capacité à tirer ou non profit des ressources technologiques et scientifiques présentes en région.

Ce travail s'appuie sur les résultats d'une recherche menée dans le cadre d'un programme Pour et Sur le Développement régional (PSDR $)^{2}$, ce travail ayant pour objectif de permettre aux décideurs, et notamment aux partenaires $^{3}$ qui ont été les nôtres au cours de cette recherche, de tenter d'évaluer le potentiel en matière d'innovation des entreprises

1. «L'ancrage territorial des activités industrielles et technologiques, résulte de la conjonction entre les aspects de proximité organisationnelle, révélateurs de la dimension industrielle intra comme interfirmes, et les aspects de proximité spatiale, sur lesquels se fonde la dimension territoriale ».

2. Dans le cadre de ce programme, ce travail a bénéficié d'un financement et a été présenté au symposium "Territoires et Enjeux du Développement Régional" à Lyon, du 9 au 11 mars 2005. 3. ARIA : Association régionale des industries agroalimentaires ; ANVAR : Agence nationale de valorisation de la recherche ; CRITT : Centre régional d'innovation et de transfert de technologie ; Bourgogne Réseau : Réseau de diffusion technologique. 
agroalimentaires et le rôle des dispositifs existant au niveau régional en termes d'aide au transfert de technologie et à l'innovation. Nous proposons en fait dans cet article de répondre aux questions suivantes :

- quelles sont les conditions, en particulier en matière d'organisation, qui permettent aux entreprises agroalimentaires implantées en Bourgogne «d'absorber » les ressources de leur environnement et de développer des innovations?

- sont-elles toutes en mesure de se saisir de la même manière des opportunités présentes?

Après avoir développé dans une première partie l'analyse théorique sur laquelle nous nous appuyons pour mener ce travail, nous tenterons ensuite de répondre aux questions sur la gestion de l'innovation dans les entreprises, en nous basant sur une analyse de données élaborée à partir d'enquêtes réalisées dans 41 entreprises agroalimentaires localisées en Bourgogne.

\section{Innovation et mobilisation des ressources technologiques externes}

\section{Nouvelle conception de l'innovation et " capacité d'absorption " des entreprises}

Contrairement à la vision standard de la technologie dans laquelle elle est considérée comme étant un ensemble d'informations facilement appropriables, un certain nombre de développements théoriques récents en économie de l'innovation (Rosenberg, 1976 ; Dosi, 1988 ; Dosi et al, 1990) insiste sur la construction de la technologie à l'intérieur de la firme. Cette conceptualisation de la technologie permet de prendre en compte l'importance des procédures de coordination dans la firme, et entre la firme et les autres acteurs de son environnement. Alors que le modèle linéaire de l'innovation distinguait les différents stades de recherche, conception, fabrication et commercialisation, le modèle de la chaîne interconnectée ou modèle de liaison en chaîne (Kline et
Rosenberg, 1986) prend en compte les feedback et les interactions entre les services de recherche, de conception, de fabrication et de commercialisation qui permettent de redéfinir, si besoin, l'innovation en fonction des contraintes qui peuvent survenir aux différents stades. En effet, l'échange d'informations entre individus intervenant dans différentes activités permet non seulement de résoudre les problèmes techniques qui apparaissent aux différents moments et stades du processus d'innovation, mais il autorise aussi les individus à tenir compte d'informations inconnues au début du processus afin de commercialiser un produit ou un procédé conforme aux attentes et besoins des clients.

Le processus d'apprentissage est alors le processus qui va permettre à une firme d'acquérir des connaissances ou des savoirs et des savoir-faire spécifiques (Le Bas, 1993 ; Foray, 1994). La production de nouvelles connaissances peut être intentionnelle et concerner les laboratoires de R\&D. L'acquisition de connaissances peut être également considérée comme le produit-joint d'autres activités, comme les activités de fabrication par exemple. L'apprentissage comme facteur décisif de la maîtrise des technologies dans une firme n'est pas totalement un thème nouveau en économie. Arrow avait ainsi élaboré en 1962 le concept d'apprentissage par la pratique learning by doing, cet apprentissage se traduisant par une amélioration des performances des travailleurs et une augmentation de la productivité. Dans les années 1980, Rosenberg (1982) et Lundvall (1988) ont enrichi cette analyse en proposant de prendre en compte les améliorations et, plus globalement, les développements technologiques se produisant au cours de l'utilisation learning by using et grâce aux interactions entre producteurs et utilisateurs de technologies learning by interacting. Dosi et al, op. cit.) ont affirmé que l'apprentissage en jeu dans l'appropriation des technologies n'était pas 
uniquement technologique mais également organisationnel dans la mesure où il va dépendre du contexte organisationnel dans lequel il se développe.

En raison de leur rôle fondamental dans le processus d'innovation, la manière dont les processus d'apprentissage technologique sont gérés, c'est-à-dire dont ils sont favorisés ou inhibés, apparaît comme un des facteurs explicatifs de la plus ou moins grande capacité des firmes à innover. Les processus d'apprentissage dépendent notamment des mécanismes de coordination dans la firme et des relations qu'elle entretient avec des acteurs externes comme des universités ou d'autres firmes. La capacité d'une firme à développer un double processus d'assimilation de la connaissance technologique externe et de création de nouvelles compétences détermine pour Cohen et Levinthal $(1989,1990)$ sa « capacité d'absorption». L'idée de ces auteurs est que la capacité d'une firme à exploiter les connaissances externes est un déterminant critique de sa capacité d'innovation : une firme sera plus ou moins en mesure d'exploiter les opportunités technologiques de son environnement selon son savoir de base et le processus d'apprentissage qui s'effectue en son sein. Les dépenses de R\&D déployées représentent, selon eux, un indicateur de la capacité d'absorption d'une entreprise dans la mesure où le département de R\&D a un double rôle : celui d'innover, de créer de nouvelles connaissances, mais aussi, celui de permettre à la firme de suivre les évolutions et d'anticiper les opportunités technologiques. Si la connaissance externe est éloignée de sa base de connaissance existante, l'entreprise devra en effet fournir des efforts importants pour pouvoir l'exploiter et elle aura, de plu,s de grandes difficultés à déceler la valeur de cette connaissance. Cependant accroître la capacité d'absorption de la firme ne se limite pas à investir dans la R\&D. Conformément au schéma de Kline et Rosenberg, l'exploitation d'une opportunité technologique à l'intérieur de l'entreprise exige que des transferts de connaissance aient lieu entre les sous-unités de l'organisation. La capacité d'absorption dépend donc de manière primordiale des modalités organisationnelles d'échange d'informations et de connaissances qui permettront aux individus dans une firme d'exploiter une opportunité technologique.

Ce concept même s'il semble proche de celui d'externalités technologiques (ou captation des spills overs) repose sur une conception très différente de la firme et de la manière dont elle conduit ses projets d'innovation. En effet si dans ces deux perspectives, la capacité d'une firme à exploiter les connaissances externes est un déterminant critique de sa capacité d'innovation, la référence au concept de capacité d'absorption insiste sur le fait qu'une firme sera plus ou moins en mesure d'exploiter les opportunités technologiques de son environnement selon son savoir de base et le processus d'apprentissage qui s'effectue en son sein. Ces compétences sont construites au cours du temps et des expériences et conditionnent la capacité des firmes à tirer parti c'est-à-dire à détecter et exploiter les connaissances externes.

Dans cette perspective, l'assimilation des connaissances technologiques externes n'est pas entendue comme un recours mécanique et linéaire à des informations technologiques produites dans l'environnement scientifique des entreprises. Le concept de capacité d'absorption indique au contraire que ce recours à l'environnement technologique doit se comprendre comme un processus complexe, incertain, cumulatif, fortement interactif, voire coopératif, d'acquisition des ressources d'innovation et de constitution d'apprentissages collectifs. La reconnaissance de ces interactions entre les firmes et leur environnement technologiques conduit de nombreux auteurs (Axelsonn et Easton, 1992 ; Gay et Picard, 2001), à envisager une 
organisation particulière pour l'acquisition des ressources d'innovation : les réseaux d'innovation.

On peut évoquer deux approches des réseaux particulièrement intéressantes pour illustrer notre propos.

- La première, en termes de réseaux technico-économiques, a été développée par des sociologues tels que Akrich et al, (1988) ou Callon (1991). Elle est centrée sur l'innovation et cherche à analyser le processus collectif réticulaire qui a permis l'émergence et la « diffusion » de cette innovation. Celle-ci résulte de la mise en œuvre de capacités cognitives et de capacités relationnelles des acteurs : la nature des rapports hiérarchiques, les formes de négociation et de résolution des conflits, ou les formes de coopération entre acteurs, structurent l'espace de création et de transfert des connaissances. Cependant, notre démarche, centrée sur l'entreprise et son organisation et non sur des innovations spécifiques en tant que telles, s'intègre plutôt dans des analyses qui donnent à la notion de réseau une conception plus large (Lundvall, op. cit.). Les réseaux technologiques peuvent être définis comme "l'ensemble des relations volontairement tissées par les firmes avec leur environnement, relations interactives qui dépassent la figure des relations bilatérales et visent à accroître leur capital technologique (matériel, immatériel, humain)» (Le Bas et al., 1998). Le postulat de base est que les partenaires sont mutuellement dépendants des ressources contrôlées par l'autre et que chacun aura intérêt à une mise en commun. La notion du learning by interacting (Lundvall, op. cit.) débouche sur une conception des réseaux comme structure d'apprentissage collectif. Bien qu'encadrés par un jeu d'institutions le plus souvent nationales (et composant le Système national d'innovation) (Freeman, 1988), voire régionales (Système régional d'innovation), le processus d'innovation et les réseaux qui structurent ce processus n'apparaissent pas comme localisés : les accords de coopération sont ainsi établis au niveau national, voire international.

- La seconde approche à laquelle nous pouvons nous référer est celle qui a trait aux analyses en termes de districts industriels dans la lignée des travaux de Marshall (Becattini, 1992) ou en termes de milieux innovateurs (Aydalot, 1986 ; Maillat, 1995). Ces travaux mettent en évidence l'importance du contexte socio-territorial dans l'émergence d'innovations. L'appartenance des entreprises à un même contexte socioterritorial permet de tisser des liens de confiance et de coopération basés sur des règles et valeurs communes. Cette approche met l'accent sur la constitution d'ensembles industriels délimités par un territoire et sur le rôle de la proximité géographique entre acteurs. Notre perspective consistera, dans cette mouvance, à étudier l'articulation qui existe entre des relations locales et globales, ainsi qu'entre différentes formes de proximité (Zimmermann, 1998).

\section{La proximité : une dimension incontournable des réseaux $d$ 'innovation}

Lorsque l'on s'intéresse aux relations qu'établissent les PME avec leur environnement dans le cadre d'une région, la question du rôle de la proximité (Rallet et Torre, 2004) se pose avec acuité. En effet de nombreux travaux économiques indiquent que la proximité géographique favorise le transfert de technologies. Par ailleurs, la politique de transfert technologique est mise en œuvre essentiellement à un niveau régional à travers les contrats de plan et la mise en place de structures régionales de prospection des entreprises. Tout semble indiquer que la proximité géographique des acteurs est une condition nécessaire au transfert de technologie.

Quel est le rôle joué par la proximité des « fournisseurs» de technologies pour « absorber» des connaissances externes et innover? Des entreprises appartenant au 
même secteur (ici l'agroalimentaire) et situées sur un même territoire parviennentelles à se saisir des opportunités technologiques de la même façon ? Et si ce n'est pas le cas quels sont les facteurs qui expliquent ces différences?

Généralement, la question de l'inscription spatiale des PME est assez peu traitée de manière frontale (Rallet et Torre, op. cit.). Leur analyse repose souvent sur des stéréotypes tels que l'équivalence implicite faite entre PME et relations économiques de proximité, la petite firme étant supposée attachée à son territoire et aux relations entretenues avec ses clients, fournisseurs et concurrents locaux. L'un de nos objectifs est justement de montrer que ces équivalences masquent en réalité des différences assez sensibles entre firmes du point de vue de la gestion des innovations et des relations nouées avec des acteurs externes. Dans cette optique, les analyses économiques récentes ayant trait aux systèmes localisés d'innovation reposent sur deux hypothèses complémentaires :

- la première ayant trait au rôle joué par la proximité entre les acteurs,

- la seconde tenant au caractère particulier de la connaissance et aux problèmes posés par son transfert.

En premier lieu donc, et dans la mesure où les activités d'innovation sont caractérisées par un poids important des connaissances tacites, les relations de face à face, la fréquence des relations et par conséquent une proximité géographique deviennent une condition primordiale pour que puissent se développer ces apprentissages et ces processus d'innovation. En résumé, les échanges d'informations et la création de connaissances communes aux producteurs et aux utilisateurs ne peuvent survenir que s'il règne entre les firmes d'abord un climat de confiance mutuelle, car sans cette confiance, chacun pourra craindre que l'autre partie ne se conduise de manière opportuniste. Ensuite, l'instauration de cette confiance, d'un code commun de communication et de conventions permettant de stabiliser les relations, sont des processus qui réclament du temps. La notion de proximité organisationnelle (Pecqueur et Zimmerman, 2002) développée par les économistes traitant de la question spatiale reprend cette idée qu'au-delà de la proximité géographique, le transfert de connaissances exige aussi une proximité en termes de codes de communication et de stabilisation d'un système de relations.

Si toutes les entreprises que nous avons enquêtées sont localisées en Bourgogne, leur insertion sur un territoire est multidimensionnelle et difficile à définir avec précision. Leur périmètre organisationnel est en effet souvent complexe à identifier ${ }^{4}$ et leur insertion résulte d'une construction progressive, qui tente de concilier différentes contraintes : proximité de sources d'informations et de certains partenaires mais aussi proximité des marchés, facilité par rapport à l'accès en matière de transports, proximité d'un bassin d'emploi, etc.

\section{Organisation et innovation des entreprises agroalimentaires : une typologie}

Si la proximité apparaît une dimension incontournable dans le transfert de connaissances et le processus d'innovation, il nous semble important d'enrichir cette perspective en prenant en compte d'autres dimensions des entreprises. L'une de nos hypothèses est que l'appartenance sectorielle, le positionnement marché des entreprises et leur détermination à développer un avantage concurrentiel déterminent en partie la manière dont une entreprise est organisée en interne (ses modes de relations entre services, la manière dont sont gérés les projets d'innovation) et son mode d'insertion dans

4. L'intégration d'une entreprise dans un groupe complexifie l'analyse de son organisation et de son insertion dans les réseaux. 
des réseaux. En effet, la modification des produits et des processus ne doit pas être considérée uniquement comme le signe de la capacité des entreprises à s'adapter à des modifications externes. Les entreprises, ou au moins une partie d'entre elles, cherchent à orienter la dynamique technologique et elles s'engagent dans des démarches qui pourront leur permettre de se différencier des autres entreprises et à terme leur donner une place de leader sur le marché et qui auront pour conséquence de redéfinir l'environnement économique et technologique de la firme (Gaffard, 1989). Les stratégies d'innovation et d'absorption de technologies externes sont alors intimement liées aux marchés sur lesquels les entreprises interviennent et ces dernières pensent en même temps les rapports entre technologies, produits et marchés (Le Bas, 1993). Concernant le secteur agroalimentaire, les travaux de Lambert (2001) ont montré, par exemple, le rôle tout à fait prépondérant de la capacité d'innovation pour certaines entreprises spécialisées sur le secteur des Produits alimentaires intermédiaires (PAI). En effet, face à une complexité de plus en plus grande des produits alimentaires, certains fournisseurs d'ingrédients et de composants tentent de déployer une stratégie de différenciation leur permettant de se positionner comme fournisseur exclusif d'autres entreprises qui assemblent ensuite ces produits. À l'image de secteurs comme la chimie ou l'automobile, ces entreprises développent des stratégies centrées sur l'innovation afin de proposer des produits plus complexes en fourniture spéciale et de fidéliser leurs clients.

L'hypothèse que nous avons testée dans ce travail est la suivante : la capacité à « absorber » les technologies et connaissances externes (à mobiliser les ressources régionales existantes) dépend de la manière dont l'entreprise est organisée en interne (circulation de l'information, dispositifs de veille technologique, existence de service
R\&D et/ou qualité), organisée vis-à-vis de son environnement extérieur (insertion dans des réseaux d'innovation). Cette organisation construite par apprentissage au cours du temps étant elle-même fortement dépendante du positionnement marché (clients, circuits de commercialisation), et de la stratégie de l'entreprise (stratégie d'innovation, qualité, recherche nouveaux clients).

\section{Point méthodologique : le dispositif d'enquête}

Nous avons constitué une population de 41 entreprises agroalimentaires appartenant à 22 secteurs des industries agroalimentaires de la région Bourgogne. Elles se répartissent en deux catégories :

- celles (29) qui étaient connues pour avoir eu recours, à divers titres, aux institutions de transfert bourguignonnes,

- l'autre partie (12) n'étant pas connue de Bourgogne Réseau, organisme d'aide à l'innovation et au transfert de technologies.

Nous avons mené des enquêtes directes auprès des entreprises sur la base d'un questionnaire comportant des questions ouvertes et fermées. La construction du questionnaire d'enquête a été orientée en fonction du souci que nous avions de recueillir des informations sur les caractéristiques organisationnelles des firmes, tout en ne nous écartant pas des critères d'organisation et de caractérisation de l'innovation telles qu'elles sont énoncées dans le Manuel d'Oslo (OCDE, 1997). Le choix de la population à enquêter relève :

1) de la recherche d'une représentativité satisfaisante de la structure sectorielle des Industries agroalimentaires (IAA) régionales ;

2) de la volonté de retenir des entreprises dont les métiers et le positionnement sur le marché sont différents, de comparer celles qui sont en contact avec les organismes de transfert et celles qui ne le sont pas.

Notre objectif était de mettre en évidence la diversité de potentiel d'innovation des 
entreprises en matière d'innovation en réalisant une typologie afin de dégager des comportements «types » d'entreprises. Pour ce faire, nous avons privilégié une entrée par l'organisation de l'entreprise comme support de la capacité d'innovation et tenté d'évaluer l'un des déterminants clés de l'innovation dans les entreprises des secteurs des IAA, la « capacité d'absorption » des connaissances externes. Alors que dans l'approche de Cohen et Levinthal, elle est mesurée par les investissements de R\&D, nous mobilisons d'autres indicateurs pour mesurer cette capacité et évaluer l'effort consacré par les firmes à la recherche et au développement en adoptant une vision plus conforme à la réalité des PME agroalimentaires qui ne possèdent pas de service de R\&D formalisé.

Les indicateurs retenus pour réaliser cette typologie permettent de mieux appréhender le potentiel d'innovation des entreprises.

- Les effectifs salariés affectés aux fonctions de recherche et de qualité. Dans les entreprises, surtout celles qui ne disposent pas d'un service de R\&D, nos travaux mettent en évidence le rôle central tenu par le service qualité dans la résolution des problèmes techniques. Ces problèmes peuvent être liés aux respects des normes en matière sanitaire et de traçabilité ou en réponse aux demandes d'amélioration continue du process et des produits de la part des clients industriels et de la distribution. La personne responsable de ce service occupe souvent une position de « traducteur » entre l'entreprise et son environnement, y compris dans le cadre d'un projet de collaboration et d'innovation avec un partenaire extérieur.

- La capacité de l'entreprise à se mettre en relation avec des acteurs externes. Celle-ci a elle-même été évaluée en recourant à deux indicateurs :

1) quantification des relations selon les partenaires (entreprises, clients ou fournisseurs ; organismes de recherche ; centres techniques ; écoles),
2) intensité de la relation (durée et contractualisation ou non de la relation). Pour chaque entreprise, nous avons pu ainsi établir un indicateur : il est faible quand l'entreprise a noué des relations peu formalisées sur des périodes limitées dans le temps et il est fort quand l'entreprise s'est engagée dans des relations très formalisées sur le long terme.

- Le positionnement des entreprises au sein de leur industrie ou sur leurs marchés, en mobilisant deux indicateurs : l'importance respective dans le chiffre d'affaires de l'entreprise des circuits Grandes et moyennes surfaces (GMS) et des clients industriels.

\section{Positionnement de l'entreprise sur les marchés et insertion dans les réseaux : les dimensions clés de la capacité d'innovation des entreprises}

Cinq catégories d'entreprises homogènes (voir annexe et tableau 1) du point de vue des variables actives représentatives ont été constituées en utilisant une méthode de classification automatique. La typologie permet d'ordonner la diversité des entreprises que nous avons visitées et ainsi de comprendre comment elles innovent, quels sont les problèmes qu'elles essayent ainsi de résoudre et quelles ressources (régionales et autres) elles mobilisent pour le faire.

\section{Des entreprises « traditionnelles »...}

Ces entreprises de très petites tailles sont indépendantes pour la grande majorité d'entre elles et se positionnent sur une niche de marché « haut de gamme ». Elles opèrent sur des créneaux difficilement mécanisables évitant ainsi d'être en confrontation directe avec les industries de «volume » pour lesquelles le prix est un élément essentiel de la concurrence. Elles sont très tournées vers le circuit traditionnel (grossistes, détaillants). Le potentiel interne d'innovation est très faible, en particulier en matière de R\&D. Elles se caractérisent par une ouverture sur l'extérieur très limitée à travers des rela- 
Tableau 1. Présentation synthétique des classes d'entreprises

\begin{tabular}{|c|c|c|c|c|c|}
\hline & $\begin{array}{c}\text { (1) Entreprises } \\
\text { " traditionnelles " } \\
\text { Marchés de niche, } \\
\text { nationaux, circuits } \\
\text { traditionnels }\end{array}$ & $\begin{array}{c}\text { (2) Entreprises } \\
\text { dont l'activité est } \\
\text { orientée } \\
\text { vers la région } \\
\text { hors circuit GMS }\end{array}$ & $\begin{array}{c}\text { (3) Entreprises } \\
\text { tournées vers } \\
\text { la GMS } \\
\text { Marchés de niche }\end{array}$ & $\begin{array}{c}\text { (4) Entreprises } \\
\text { se positionnant } \\
\text { sur des marchés } \\
\text { de volume } \\
\text { circuit GMS }\end{array}$ & $\begin{array}{l}\text { (5) Entreprises } \\
\text { de PAl* } \\
\text { Marchés } \\
\text { industriels }\end{array}$ \\
\hline $\begin{array}{l}\text { Effectif salarié } \\
\text { moyen }\end{array}$ & 21 & 77 & 57 & 278 & 58 \\
\hline $\begin{array}{c}\text { Potentiel } \\
\text { d'innovation }\end{array}$ & $\begin{array}{c}\text { Potentiel très } \\
\text { faible, faible } \\
\text { formalisation des } \\
\text { savoirs }\end{array}$ & $\begin{array}{l}\text { Potentiel très } \\
\text { faible }\end{array}$ & $\begin{array}{c}\text { Potentiel très } \\
\text { faible, qualité } \\
\text { très développée }\end{array}$ & $\begin{array}{l}\text { Logique de } \\
\text { groupe }\end{array}$ & Potentiel fort \\
\hline $\begin{array}{l}\text { Relations } \\
\text { externes }\end{array}$ & $\begin{array}{l}\text { Très peu de } \\
\text { relations } \\
\text { (fournisseurs et } \\
\text { écoles) }\end{array}$ & $\begin{array}{c}\text { Nombre de } \\
\text { relations dans la } \\
\text { moyenne }\end{array}$ & $\begin{array}{c}\text { Peu de relations } \\
\text { mais plus } \\
\text { formalisées }\end{array}$ & $\begin{array}{l}\text { Nombreuses } \\
\text { relations } \\
\text { formalisées }\end{array}$ & $\begin{array}{l}\text { Nombreuses } \\
\text { relations } \\
\text { formalisées }\end{array}$ \\
\hline $\begin{array}{l}\text { Localisation } \\
\text { des relations }\end{array}$ & \multicolumn{3}{|c|}{ Majoritairement liées avec les organismes de la région } & \multicolumn{2}{|c|}{ Relations hors région plus nombreuses } \\
\hline $\begin{array}{c}\text { Activités } \\
\text { d'innovation }\end{array}$ & $\begin{array}{c}\text { Faibles et } \\
\text { "routinières ». } \\
\text { Pas de capacité } \\
\text { à anticiper les } \\
\text { problèmes } \\
\text { technologiques }\end{array}$ & $\begin{array}{c}\text { Faibles et } \\
\text { "routinières ». } \\
\text { Compense leur } \\
\text { faible potentiel } \\
\text { interne par } \\
\text { l'établissement } \\
\text { de relations } \\
\text { externes }\end{array}$ & $\begin{array}{c}\text { Faibles mais plus } \\
\text { radicales. } \\
\text { Mise } \\
\text { au point } \\
\text { de procédés } \\
\text { originaux }\end{array}$ & $\begin{array}{c}\text { Fortes } \\
\text { et radicales. } \\
\text { L'objectif: } \\
\text { se différencier } \\
\text { des concurrents }\end{array}$ & $\begin{array}{c}\text { Fortes } \\
\text { et radicales. } \\
\text { Logique de co- } \\
\text { développement } \\
\text { avec les clients }\end{array}$ \\
\hline
\end{tabular}

* Produits alimentaires intermédiaires - Source : les auteurs

tions très ponctuelles et peu formalisées. L'essentiel des relations se noue avec les écoles (formant des ingénieurs ou des techniciens supérieurs) et dans une moindre mesure avec d'autres entreprises localisées très majoritairement en Bourgogne. Ces entreprises utilisent peu les réseaux institutionnels disponibles au niveau régional.

\section{...qui n'anticipent pas leurs problèmes tech-} niques

Presque toutes les entreprises mettent sur le marché des produits nouveaux pour l'entreprise. L'activité d'innovation produit de ces entreprises peut être qualifiée d'in-

5. Intégrant la modification du conditionnement, de la recette, l'adaptation du poids du produit en fonction de la demande du consommateur. crémentale ou de « routinière» $^{5}$. Le développement se fait essentiellement en interne avec un apport limité de partenaires extérieurs qui se limite pour l'essentiel aux écoles. Les innovations de procédé se résument à l'adoption de techniques déjà existantes. Le développement se réalise en faisant appel aux compétences des fournisseurs sans participation significative des entreprises à l'adaptation des machines.

Des entreprises dont l'activité est régionale (hors circuit GMS)...

Ces entreprises sont indépendantes pour la moitié d'entre elles et se sont positionnées en dehors du marché de la GMS. Ce positionnement, selon les entreprises, relève de logiques différentes provenant 
notamment de la nature de leur clientèle (agriculteur, boulanger, comité d'entreprises, etc.). L'activité est très tournée vers la région pour ces entreprises de taille proche de la moyenne. Elles produisent généralement sous signe de qualité et/ou d'origine contrôlée. Elles ont recours plus souvent que les entreprises « traditionnelles » à des ressources extérieures localisées majoritairement dans la région, y compris aux organismes de transfert technologique ou à des laboratoires de recherche publique, mais ces relations restent le plus souvent informelles.

... qui compensent en partie leur faible potentiel interne d'innovation par l'établissement de relations externes

La quasi-totalité des entreprises mettent sur le marché des produits nouveaux pour l'entreprise et proposent peu de produits nouveaux pour le marché. Les entreprises réalisent des innovations de procédé en adoptant des techniques déjà existantes. Cependant au sein de certaines entreprises existe également la mise au point de procédés originaux. Le développement se fait en interne avec un apport de partenaires extérieurs plus important et diversifié que pour les entreprises « traditionnelles », même si ces relations restent ponctuelles et non formalisées.

Des entreprises dont les clients GMS sont localisés hors de la région...

Ces entreprises forment avec les entreprises « traditionnelles » un continuum en termes de logiques organisationnelles. Elles sont de petites tailles et indépendantes. Elles se situent sur un marché de niche, mais leur activité est orientée vers les marchés nationaux en utilisant plus souvent le circuit GMS. Une entreprise sur deux fabrique des marques de distributeurs (MDD). Elles essayent de maintenir un équilibre entre leurs divers cir- cuits de commercialisation dans le souci de ne pas être trop dépendantes de la GMS et ainsi mieux valoriser leurs productions dont une part significative bénéficie de signe de qualité et/ou d'appellation d'origine contrôlée.

Ces entreprises ont fait un choix stratégique : recruter un « ingénieur qualité ». Ce choix est motivé par la nécessité de faire évoluer l'organisation afin qu'elle soit en cohérence avec le souhait d'élargir leurs débouchés vers la GMS. Les entreprises sont peu ouvertes vers l'extérieur mais les relations qu'elles nouent avec les écoles, les entreprises ou les institutionnels du transfert sont plus formalisées et de durée plus longue que les entreprises « traditionnelles ». La majeure partie de ces relations se réalise avec des partenaires bourguignons. La mise en concurrence des fournisseurs reste marginale, les entreprises privilégient les relations de long terme avec les partenaires sur la base de relations interpersonnelles.

...dont l'activité d'innovation est orientée vers l'automatisation du processus de production L'activité d'innovation est faible quantitativement en produits et radicale si l'on considère qu'une majorité d'entreprises déclarent avoir commercialisé des produits nouveaux pour le marché. Elle est forte et radicale en procédé. Les entreprises de cette catégorie parviennent à engager des innovations radicales en produits et en procédés parce qu'elles possèdent une capacité qui leur permet de trouver et de mobiliser des compétences externes. La présence d'un ingénieur qualité leur permet de compenser en partie l'absence d'un service de R\&D. Leur métier est à l'origine artisanal avec une production de petites séries sous signe de qualité. Les innovations-procédés tendent à faciliter le processus d'automatisation de leur production qu'elles ont engagé tout en maintenant un niveau élevé de qualité gustative et bactériologique. 
Des entreprises qui se positionnent sur des marchés de volume...

Ces grandes entreprises ${ }^{6}$ sont majoritairement intégrées dans un groupe, elles commercialisent leur production sur les marchés nationaux et internationaux via une politique systématique de référencement auprès des principales centrales d'achat du circuit GMS. Leur production est à destination des marchés de « volume » où la concentration de la production est forte et où la concurrence s'exprime à travers la possession de marques leaders dont la notoriété s'appuie sur des dépenses de publicité élevées. La production pour le compte des distributeurs représente une activité non négligeable. Les données d'organisation de ces entreprises sont à prendre avec précaution. En effet la structure groupe complique l'analyse. Ainsi certaines fonctions comme la recherche et/ou le service commercial peuvent être localisées dans d'autres entreprises du groupe. Malgré ces réserves, nous pouvons évaluer le potentiel interne de $R \& D$ et de qualité à un niveau moyen.

Ces entreprises sont bien insérées dans les réseaux de relations majoritairement situés hors de la région et l'intensité de ces relations est moyenne. Elles recourent à toutes les compétences externes que ce soit les centres de transfert technologique, les écoles ou les organismes de recherche au niveau régional et hors région : ceci est lié à leur potentiel interne d'innovation plus développé si l'on prend en compte le niveau groupe. Les relations avec les fournisseurs sont formalisées et passent par l'établissement de cahiers des charges et la mise en concurrence. Cette catégorie se distingue donc des autres dans le cadre de ces relations avec les fournisseurs : la taille des entreprises permet de parler d'égal à égal avec les

6. L'effectif salarié moyen de ces entreprises est important (278 salariés) comparativement à l'effectif moyen dans les autres classes qui oscille entre 21 et 77 . fournisseurs. La logique de ces entreprises est davantage la recherche de baisse des coûts et donc de mise en concurrence des fournisseurs alors que les autres catégories privilégient des relations de long terme avec ceux-ci.

...dont l'activité d'innovation s'inscrit avec la publicité comme un moyen d'asseoir la marque

L'activité d'innovation est forte et radicale en produits. L'activité d'innovation est en cohérence avec la stratégie globale de ces entreprises qui se positionnent sur des marchés de marque. La survie d'une marque sur ce type de marché dépend étroitement des moyens consacrés à la publicité mais aussi à l'innovation. Il est impératif pour ces entreprises de mettre sur le marché de nouveaux produits afin de se différencier des concurrents et d'essayer de segmenter le marché.

Le développement des innovations se fait en interne sous la responsabilité du service de $R \& D$ qui est capable de gérer les projets d'innovation des entreprises du groupe et d'identifier et d'aller chercher les compétences externes qui lui manquent. L'innovation-procédé semble moins développée, les entreprises recourant souvent à l'achat de machines standards.

\section{Des entreprises fabriquant des produits ali- mentaires intermédiaires...}

Ces entreprises de petites tailles opèrent sur le circuit industriel, c'est-à-dire qu'elles vendent à d'autres industriels les produits alimentaires intermédiaires qui entrent dans la composition de leurs produits. Elles ne s'adressent donc pas au consommateur final. Leurs marchés sont nationaux et internationaux. Le potentiel interne de ces entreprises est important aussi bien en termes de $R \& D$ que de qualité. Elles sont très intégrées dans des réseaux localisés majoritairement hors de la région et elles ont développé des formes de relations formalisées sur 
le long terme (filiales communes, relations privilégiées avec les services de R\&D des autres entreprises, relations formalisées avec des laboratoires de recherche publique). Elles utilisent principalement les compétences des écoles d'ingénieurs, des centres de transfert technologique et des organismes de recherche. Les relations de ces entreprises avec leurs clients peuvent s'apparenter, comme le suggère Lambert (1997), au modèle de relations bien connu dans l'industrie automobile entre les constructeurs et leurs sous-traitants qui met au premier plan les collaborations entre clients et fournisseurs pour développer de nouveaux produits et procédés. Dans ce cadre, le donneur d'ordre impose souvent au sous-traitant d'être « prescripteur d'innovation » et, par conséquent, d'investir dans le domaine de la R\&D.

\section{...dont l'innovation radicale est un objectif central}

L'activité d'innovation est marquée par la place centrale qu'occupe l'innovation radicale, c'est-à-dire nouvelle pour le marché. Ces entreprises mettent des produits intermédiaires à la disposition des entreprises qui les intègrent dans leurs produits. $\mathrm{La}$ concurrence entre les entreprises productrices de PAI se situe dans leur capacité à mettre sur le marché des produits nouveaux.

\section{Quelle mobilisation des ressources technologiques régionales par les entreprises agroalimentaires bourguignonnes ?}

La moitié des relations tissées par les entreprises localisées en Bourgogne le sont avec d'autres entreprises ou institutions bourguignonnes. Il n'y a pas de différence notable de comportement entre les entreprises connues de l'organisme de prospection bourguignon et les autres. Il semble donc que la proximité (si l'on accepte de considérer la localisation régionale comme un indicateur de proximité) importe. La dimension « réseau régional » tient un rôle central dans la mise en relation des PME ayant un potentiel de R\&D faible, voire inexistant, avec les centres de compétences techniques. Le maillage institutionnel (CCI, Anvar, Aria, Bourgogne réseau, etc.) ${ }^{7}$ au niveau de la région permet aux entreprises de compenser la faiblesse de leur capacité à nouer des liens externes.

Si globalement, les entreprises agroalimentaires bourguignonnes se tournent autant vers des entreprises ou des institutions de la région et hors région, nous constatons malgré tout une différence de comportements selon les classes d'entreprises. Les entreprises peu dotées en potentiel interne sont majoritairement liées avec les organismes de la région. L'ouverture hors région se développe dès que le potentiel interne s'accroît. Les entreprises dont le potentiel interne est important développent davantage des relations hors région, en particulier celles qui produisent des PAI.

Nous distinguons deux schémas de constitution de réseaux dans la perspective d'innover.

- Les entreprises qui disposent d'un très faible potentiel interne d'innovation, ont des difficultés pour identifier leurs problèmes et trouver des compétences extérieures pour les résoudre. Elles n'ont pas ou peu de relations avec la recherche et les organismes de transfert parce qu'elles ne sont pas capables d'établir une relation avec eux et/ou de transférer les connaissances dans leur organisation. La proximité géographique ne facilite pas, dans ce cas, le transfert technologique faute de proximité organisationnelle. Elles rencontrent de nombreux obstacles lorsqu'il s'agit de mener à bien des innovations radicales. En revanche, elles développent des innovations « routinières » et font appel à cet effet aux entreprises et écoles qui parlent le «même langage » qu'elles (Albert et al, 2006). Les

7. Pour les sigles, $c f$. la note 3. 
écoles leur apportent une aide permettant de résoudre des problèmes non solubles en interne en compensant en partie la faiblesse du potentiel interne. Il faut noter que 10 entreprises parmi les 12 qui n'ont pas été en contact avec le réseau bourguignon de prospection appartiennent aux classes 1,2 ou 3 et se caractérisent, par conséquent, par un très faible potentiel d'innovation.

- Les entreprises qui possèdent un potentiel interne leur permettant de se mettre en relation avec l'ensemble des partenaires externes. La proximité géographique amplifie dans ce cas les effets de la proximité organisationnelle.

\section{Conclusion}

La capacité des entreprises agroalimentaires bourguignonnes à se mettre en relation avec d'autres entreprises ou organismes dans la perspective d'innover est déterminée avant tout par leur potentiel interne de R\&D, de leur qualité et leur organisation.

Nos résultats mettent en lumière plusieurs faits stylisés qui structurent le tissu productif agroalimentaire bourguignon. La nature des marchés sur lesquels opèrent les entreprises induit des contraintes en terme d'organisations interne et externe et des formes de concurrence différentes. Globalement les entreprises qui ont un potentiel interne d'innovation réduit, mobilisent peu et ponctuellement des ressources externes. L'absence de potentiel interne empêche l'établissement de relations formalisées et de longue durée, mais n'interdit pas la mise en place de relations ponctuelles et non formalisées. Ce type de relation est adapté pour des entreprises qui innovent de façon « routinière » en produit et qui adoptent les technologies standards extérieures.

Les entreprises qui se positionnent sur des marchés de volume ou qui produisent des PAI savent trouver les compétences externes qu'elles souhaitent mobiliser et formalisent ces relations. Les entreprises fabriquant des PAI se distinguent des autres entreprises par le niveau élevé de leur potentiel interne et les formes de relations avec l'extérieur très fréquentes, inscrites dans la durée et très formalisées. Les entreprises de ces deux classes ont une activité d'innovation plus intense en produits. La fonction première au sein du système productif agroalimentaire des entreprises qui fabriquent des PAI est de fournir aux autres entreprises des produits innovants. Elles sont donc tenues plus que les autres, pour survivre, d'innover en permanence.

Les entreprises qui ne possèdent pas de capacité de R\&D et qui n'anticipent pas leurs futurs besoins technologiques (dispositifs de veille technologique, partenariat de R\&D avec des entreprises ou des organismes de recherche) ont généralement de grandes difficultés à collaborer de manière fructueuse avec les centres de transfert technologique. Elles se plaignent pour certaines du décalage entre la proposition technologique faite par le centre et la réalité organisationnelle de l'entreprise, ce qui rend cette proposition inapplicable en l'état au sein de l'entreprise. La proximité géographique des centres de transfert n'améliore pas cette distorsion entre le projet et l'attente de l'entreprise. La collaboration avec des organismes de recherche est également complexe dans la mesure où les entreprises éprouvent cette même incapacité à construire un code commun de communication et qu'elles adoptent des horizons de temps sensiblement différents des centres de recherche. Face à un problème technologique, une entreprise agroalimentaire aura tendance à réclamer une réponse immédiate ou quasi immédiate, ce que l'organisme de recherche n'est pas en mesure généralement de fournir. Le manque d'anticipation de ces problèmes et, de façon concomitante, l'absence de proximité organisationnelle vis-à-vis des acteurs susceptibles de les aider à trouver des solutions, expliquent les difficultés à innover des PME. 
La proximité organisationnelle apparaît comme un facteur essentiel dans la mise en relation des entreprises avec d'autres entreprises et centres scientifiques et techniques. Nous ne négligeons pas, pour autant, le rôle de la proximité géographique qui, à potentiel égal, est un élément qui peut faciliter le transfert des connaissances en particulier grâce à l'action du réseau institutionnel régional de transfert technologique. Cependant, une entreprise, même si elle est proche géographiquement des centres de ressources technologiques, ne pourra réellement en bénéficier totalement que si elle a construit un certain nombre d'apprentissages et de relations avec ces partenaires externes et que si elle dispose en interne d'une capacité à « absorber » ces connaissances.

\section{RÉFÉRENCES BIBLIOGRAPHIQUES}

Akrich M., Callon M., Latour B. (1988). A quoi tient le succès des innovations. $1^{\text {er }}$ épisode : l'art de l'intéressement, Annales des Mines, juin, p. 4-17. $2^{\mathrm{c}}$ épisode : l'art de choisir les bons porte parole, Annales des Mines, septembre, p. 14-29.

Aydalot P., (ed.) (1986). Milieux innovateurs en Europe, Paris, GREMI.

Albert P., Martin M., Tanguy C. (2006). Le rôle des écoles dans le processus d'innovation : l'exemple des réseaux constitués entre les entreprises laitières et les ENIL, (École Nationale des Industries Laitières). Espaces et Sociétés $\mathrm{n}^{\circ} 125$, avril, p. 111129.

Arrow K.-J. (Juin 1962). The Economic Implications of Learning by Doing. Review of Economic Studies, n ${ }^{\circ} 29$, p. 155-173.

Axelsonn B., Easton G. (1992). Industrial Networks: a New View of Reality, New York, Routledge.

Becattini G. (1992). Le district marshallien : une notion socio-économique. In Benko G., Lipietz A. (eds.). "Les régions qui gagnent : districts et réseaux, les nouveaux paradigmes de la géographie économique ». Paris, PUF, p. 35-55.

Callon M. (1991). Réseaux technicoéconomiques et irréversibilités. In Boyer R., Chavance B., Godard O. (dir.). «Les figures de l'irréversibilité en économie ». Paris. Editions de l'Ecole des Hautes Etudes en Sciences Sociales, p. 195-230. Cohen W.M. et Levinthal D.A. (1989).
Innovation and learning: the two faces of R\&D. Economic Journal, Vol. 99, septembre, p. 569-596.

Cohen W.-M., Levinthal D.A. (1990). Absorptive capacity: a new perspective on learning and innovation, Administrative Science Quaterly, (35), p. 128-152.

Dosi G. (1988). Sources, procedures and microeconomics effects of innovation. Journal of Economic Literature, Vol. 26, p. 1120-1171.

Dosi G., Teece D., Winter S. (1990). Les frontières des entreprises vers une théorie de la cohérence de la grande entreprise. Revue d'Economie Industrielle, (51), p. 238-254.

Foray D. (1994). Les nouveaux paradigmes de l'apprentissage technologique. Revue d'Économie Industrielle, (69), p. 93-104.

Freeman C. (1988). Japan: a new national system of innovation. In Dosi G. et al. (eds.) London and New-York. Technical change and economic theory, Pinter Publishers, p. 330-348.

Gaffard J.-L. (1989). Marchés et organisation dans les stratégies technologiques des firmes industrielles. Revue d'Économie Industrielle, $\mathrm{n}^{\circ} 48$, p. 35-51.

Gay C., Picard F. (2001). Géographie des Relations Externes des Entreprises Innovantes: une étude statistique des entreprises Rhône-Alpines. Revue d'Économie Régionale et Urbaine, (5), p. 763-784. Kline S., Rosenberg N.(1986). An overview 
of innovation. In Landau R., Rosenberg N. (eds), The positive sum strategy, Washington, The National Academy Press, p. 275-305.

Lambert A. (1997). Des assembleurs et des fournisseurs de composants: nouvelle structuration dans la transformation alimentaire. Industries Alimentaires et Agricoles, p. 781-784.

Lambert A. (2001). La désintégration verticale: une réponse aux exigences de flexibilité dans les industries alimentaires, Gestion 2000, janvier-février, p. 59-78.

Le Bas C., 1993. La firme et la nature de l'apprentissage, Economie et Sociétés, Série Dynamique Technologique et Organisation, (1), $\mathrm{n}^{\circ}$ 5, p. 7-24.

Le Bas C. (1993). Le comportement d'investissement en ressources technologiques externes et l'absorption technologique dans les moyennes entreprises industrielles. Revue Internationale des PME, Vol. 6, $\mathrm{n}^{\circ}$ 2, p. 35-64.

Le Bas C., Picard F., Suchecki B. (1988). Innovation Technologique, Comportement de Réseaux et Performances : une Analyse sur Données Individuelles. Revue d'Economie Politique, 108 (5), septembreoctobre, p. 625-644.

Lundvall B. (1988). Innovation as an interactive process. In Dosi G. and al. (eds.), «Technogical Change and Economic Theory », London, Pinter Publishers, p. 349-369.
Maillat D. (1995). Milieux innovateurs et dynamique territoriale. In Rallet A., Torre A. (eds.). "Economie industrielle et économie spatiale », Paris, Economica, p. 211-231.

OCDE (1997). Manuel d'Oslo, principes directeurs proposés pour le recueil et l'interprétation des données sur l'innovation technologique, EUROSTAT, 142 p.

Pecqueur B., Zimmermann J. B. (2002). Les fondements d'une économie de proximités. Document de travail $n^{\circ}$ 02A26, GREQAM, UMR-CNRS 6579, EHESS, Université Aix-Marseille II et III, 23 p.

Rallet A., Torre A. (2001). Proximité géographique ou proximité organisationnelle? Une analyse spatiale des coopérations technologiques dans les réseaux localisés d'innovation. Économie Appliquée, tome LIV, ${ }^{\circ} 1$, p. 147-171.

Rallet A., Torre A. (2004). Proximité et localisation. Économie rurale, $\mathrm{n}^{\circ} 280$, mars-avril, p. 25-41.

Rosenberg N. (1976). Perspectives on technology. Cambridge, University Press Cambridge.

Rosenberg N. (1982). Inside the black box: Technology and Economics, Cambridge, Cambridge University Press.

Zimmermann J.-B. (1998). Firmes et territoires : du nomadisme à l'ancrage territorial. In Bellet M. et al. (coord.), «Approches multiformes de la proximité ». Paris, Hermès, p. 269-287. 


\section{ANNEXE}

Pour constituer notre typologie, nous avons eu recours à un programme d'analyse en composantes principales couplée à une classification hiérarchique. La classification va définir à partir des axes factoriels retenus des classes d'entreprises homogènes. Le principe d'agrégation des entreprises est basé sur le principe de la recherche à chaque étape de la partition de la variance interne à la classe minimale, et par conséquent une variance maximale entre classes. Cette méthode tend à constituer des groupes d'entreprises en minimisant la variance interne ${ }^{8}$ à la classe et en maximisant la variance entre les classes.

\section{Description des classes}

\begin{tabular}{|c|c|c|c|c|c|c|c|}
\hline \multicolumn{2}{|c|}{ Variables } & \multirow{2}{*}{$\begin{array}{l}\text { \% effectifs } \\
\text { R\&D } \\
1,15\end{array}$} & \multirow{2}{*}{$\begin{array}{l}\text { \% effectifs } \\
\text { qualité } \\
3,56\end{array}$} & \multirow{2}{*}{\begin{tabular}{|l|l}
$\begin{array}{l}\text { Nombre de } \\
\text { relations }\end{array}$ \\
5,65 \\
\end{tabular}} & \multirow{2}{*}{$\begin{array}{l}\begin{array}{l}\text { Intensité } \\
\text { des relations }\end{array} \\
2,07\end{array}$} & \multirow{2}{*}{$\begin{array}{l}\begin{array}{l}\text { Circuit } \\
\text { GMS }\end{array} \\
30,88\end{array}$} & \multirow{2}{*}{$\begin{array}{l}\begin{array}{l}\text { Circuit } \\
\text { industrie }\end{array} \\
16,39\end{array}$} \\
\hline Classes & $\begin{array}{l}\text { Moyenne } \\
\text { générale }\end{array}$ & & & & & & \\
\hline 1 & $\begin{array}{l}\text { V test }{ }^{9} \\
\text { Probabilité } \\
\text { Moyenne }\end{array}$ & & & $\begin{array}{l}-3,24 \\
0,001 \\
3,33 \\
\end{array}$ & $\begin{array}{l}-3,10 \\
0,001 \\
1,43\end{array}$ & & \\
\hline 2 & $\begin{array}{l}V \text { test } \\
\text { Probabilité } \\
\text { Moyenne }\end{array}$ & & $\begin{array}{l}-2,35 \\
0,009 \\
1,83\end{array}$ & & & $\begin{array}{l}-3,58 \\
0,000 \\
2,77\end{array}$ & \\
\hline 3 & $\begin{array}{l}\text { test } \\
\text { Probabilité } \\
\text { Moyenne }\end{array}$ & & $\begin{array}{l}4,30 \\
0,000 \\
8,30\end{array}$ & \begin{tabular}{|l|}
2,43 \\
0,008 \\
7,60 \\
\end{tabular} & & & \\
\hline 4 & $\begin{array}{l}\text { test } \\
\text { Probabilité } \\
\text { Moyenne }\end{array}$ & & & & & $\begin{array}{l}4,64 \\
0,000 \\
74,50 \\
\end{array}$ & \\
\hline 5 & $\begin{array}{l}\mathrm{V} \text { test } \\
\text { Probabilité } \\
\text { Moyenne }\end{array}$ & $\begin{array}{l}4,57 \\
0,000 \\
5,86\end{array}$ & & \begin{tabular}{|l|}
2,43 \\
0,008 \\
7,60
\end{tabular} & $\begin{array}{l}3,39 \\
0,000 \\
2,84\end{array}$ & & $\begin{array}{l}5,35 \\
0,000 \\
95,00\end{array}$ \\
\hline
\end{tabular}

Source : les auteurs

8. La méthode d'agrégation est fondée sur l'utilisation du critère de perte d'inertie minimale dit critère de Ward généralisé.

9. Lorsque la valeur-test est supérieure à 2 en valeur absolue, un écart est significatif au seuil usuel (5\%). 